\title{
A genetically encoded probe for cysteine sulfenic acid protein modification in vivo
}

Christina L. Takanishi ${ }^{1}$, Li-Hua Ma ${ }^{1}$ \& Matthew J. Wood ${ }^{1 *}$

1 Department of Environmental Toxicology, University of California, Davis, CA 95616.

\section{Supporting Information}

\section{Materials and Methods}

The protein sequences for BtuE (Accession number: P06610), Gpx1 (Accession number: NP_012899), Gpx2 (Accession number: NP_009803) and Orp1/Gpx3 (Accession number: P4O581) were obtained from the NCBI Entrez Protein database. The sequences were aligned with ClustalW (http://www.ebi.ac.uk/Tools/clustalw/index.html) using the default parameters.

Supplemental Figure 1. Sequence alignment of BtuE and glutathione peroxidases from S. cerevisiae. BtuE was aligned against Gpx1, Gpx2 and Orp1/Gpx3 using CLUSTALW. The conserved cysteine residues are shown in yellow. Strictly conserved residues are indicated with an asterisk and partially conserved residues are indicated with a semi-colon. BtuE contains an insertion from residues Pro116 to Asp122 that corresponds to the human Gpx tetramerizaiton motif. 


\section{Takanishi, et al. Supplemental Figure 1}

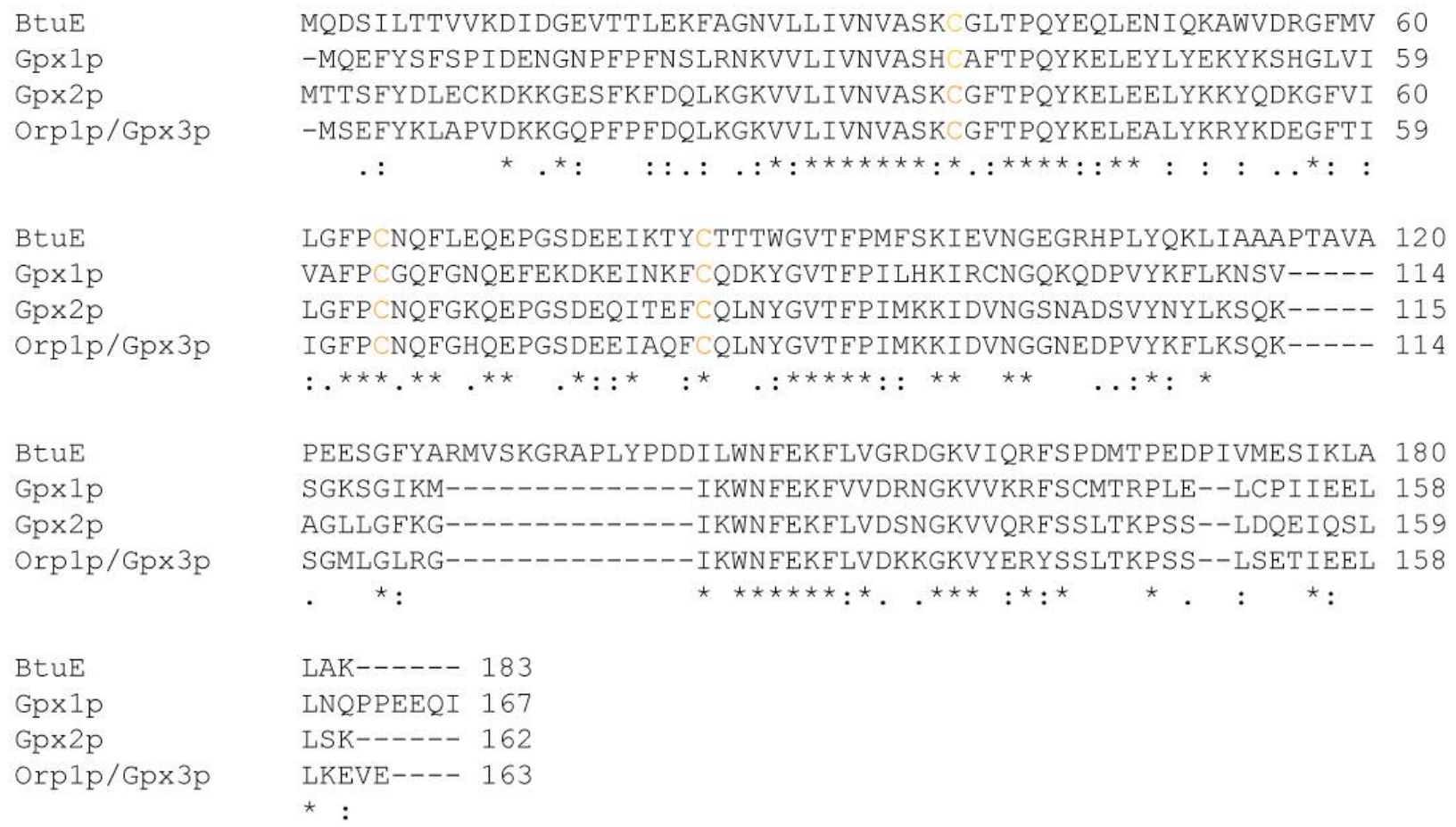

